\title{
EXISTENCE AND NONEXISTENCE OF RADIAL POSITIVE SOLUTIONS OF SUPERLINEAR ELLIPTIC SYSTEMS
}

\author{
Abdelaziz Ahammou
}

Abstract

The main goal in this paper is to prove the existence of radial positive solutions of the quasilinear elliptic system

$$
\left(S^{+}\right) \begin{cases}-\Delta_{p} u=f(x, u, v) & \text { in } \Omega, \\ -\Delta_{q} v=g(x, u, v) & \text { in } \Omega, \\ u=v=0 & \text { on } \partial \Omega,\end{cases}
$$

where $\Omega$ is a ball in $\mathbf{R}^{N}$ and $f, g$ are positive continuous functions satisfying $f(x, 0,0)=g(x, 0,0)=0$ and some growth conditions which correspond, roughly speaking, to superlinear problems. Two different sets of conditions, called strongly and weakly coupled, are given in order to obtain existence. We use the topological degree theory combined with the blow up method of Gidas and Spruck. When $\Omega=\mathbf{R}^{N}$, we give some sufficient conditions of nonexistence of radial positive solutions for Liouville systems.

\section{Introduction and main results}

We are concerned with the existence of radial positive solutions of the problem

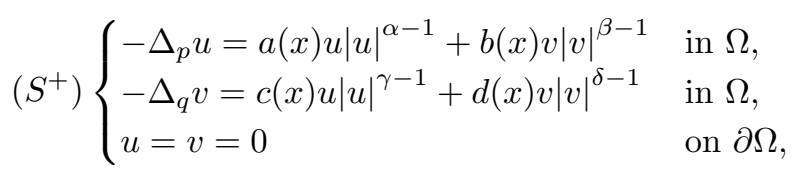

where $\Omega:=B_{R}$ is the ball in $\mathbf{R}^{N}$ centered at zero and radius $R>0$. Here as usual for $m>1(m=p, q), \Delta_{m}$ denotes the $m$-Laplacian operator.

2000 Mathematics Subject Classification. 35J25, 35J60.

Key words. Blow up argument, topological degree theory. 
During the last years the problem of existence for $\left(S^{+}\right)$has been studied by many authors, see for example, $[\mathbf{1}],[\mathbf{2}],[\mathbf{7}],[\mathbf{8}],[\mathbf{1 3}],[\mathbf{1 4}]$, $[\mathbf{1 5}],[\mathbf{1 7}]$. In particular in $[\mathbf{1 6}]$, Souto proved the existence of a positive solution taking $\Omega$ a smooth general bounded domain in $\mathbf{R}^{N}$ and $p=$ $q=2$. In the case $p \neq q$, we mention the recent results of Boccardo, Fleckinger and de Thélin [2] where the authors prove the existence of solutions of the following problem:

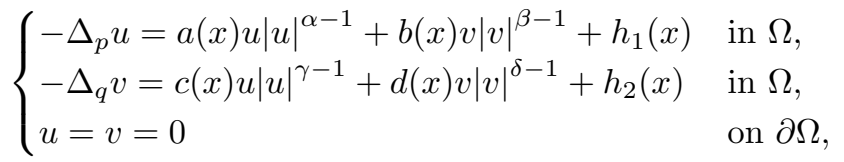

when

$$
p-1 \geq \alpha, \quad q-1 \geq \delta
$$

and

$$
(p-1)(q-1)>\beta \gamma
$$

with $\Omega$ a smooth general bounded domain in $\mathbf{R}^{N}$, and $h_{1} \in L^{p^{\prime}}(\Omega)$, $h_{2} \in L^{q^{\prime}}(\Omega)$. We remark that if $h_{1}$ and $h_{2}$ are identically zero, the solution $(u, v)$ can be a trivial solution. Our goal is to find sufficient conditions on exponents $\alpha, \beta, \gamma$, and $\delta$ in order to have a radial positive solution of $\left(S^{+}\right)$when $\Omega$ is a ball.

At this point, we introduce two subclasses of systems $\left(S^{+}\right)$:

1) System $\left(S^{+}\right)$is strongly coupled if
i) $\beta \geq \frac{q \beta+p(q-1)}{p \gamma+q(p-1)} \alpha$
and
ii) $\gamma \geq \frac{p \gamma+q(p-1)}{q \beta+p(q-1)} \delta$.

2) System $\left(S^{+}\right)$is weakly coupled if
j) $\beta<\frac{q \beta+p(q-1)}{p \gamma+q(p-1)} \alpha$
jj) $\quad \gamma<\frac{p \gamma+q(p-1)}{q \beta+p(q-1)} \delta$.

Similarly, the weakly coupled definition has been introduced in De Figueiredo [6] when j) and jj) are both satisfied. It is interesting to note that our definition includes clearly this case.

The existence and nonexistence for quasilinear systems have been studied by several authors by using different approaches; recent results can be seen in $[\mathbf{4}],[\mathbf{5}],[\mathbf{1 6}]$. For the scalar case see $[\mathbf{3}],[\mathbf{9}]$ and $[\mathbf{1 0}]$.

Now, let us make the following assumptions

$$
\max (p, q)<N
$$

$$
a, b, c, d \in C^{0}([0,+\infty[)
$$


and satisfy

$$
\inf _{r \in[0,+\infty[}(a(r), b(r), c(r), d(r))>0,
$$

$$
(p-1)(q-1)<\beta \gamma .
$$

We suppose that $\left(S^{+}\right)$is a superlinear system, i.e.,

$$
p-1<\alpha \text { and } q-1<\delta .
$$

The main results are the following.

Theorem 1.1. We assume that the system $S^{+}$is strongly coupled and that the hypotheses $\left(H_{1}\right),\left(H_{2}\right),\left(H_{3}\right)$ and $\left(H_{4}\right)$ hold. We suppose furthermore that

$\left(H_{s}\right) \max \left\{\frac{q \beta+p(q-1)}{\gamma \beta-(p-1)(q-1)}-\frac{N-p}{p-1} ; \frac{p \gamma+q(p-1)}{\gamma \beta-(p-1)(q-1)}-\frac{N-q}{q-1}\right\} \geq 0$,

is satisfied. Then, the problem $\left(S^{+}\right)$has a solution $(u, v)$ in $C^{1}\left(B_{R}\right) \cap$ $C^{2}\left(B_{R} \backslash\{0\}\right)$, such that $u>0$ and $v>0$ in $B_{R}$.

Theorem 1.2. We assume that the system $\left(S^{+}\right)$is weakly coupled and that the hypotheses $\left(H_{1}\right),\left(H_{2}\right),\left(H_{3}\right)$, and $\left(H_{4}\right)$ hold. We suppose furthermore that

$$
\left(H_{w}\right) \quad \frac{N(p-1)}{N-p}>\alpha \text { and } \frac{N(q-1)}{N-q}>\delta,
$$

is satisfied. Then the problem $\left(S^{+}\right)$has a solution $(u, v)$ in $C^{1}\left(B_{R}\right) \cap$ $C^{2}\left(B_{R} \backslash\{0\}\right)$, such that $u>0$ and $v>0$ in $B_{R}$.

We will adapt rather classical techniques of the mapping degree: we will consider the solution operator $S_{1}$ associated to a problem $\left(S^{+}\right)$acting in a suitable functional space. Then, we will look for solutions of the problem as fixed points of $S_{1}$. As it is usual in this setting, the main difficulty will be to obtain existence of a priori bounds of positive solutions.

This paper is organized as follows. Section 2 contains notations and some definitions of functional spaces and of operators $S_{\lambda}$ and $T_{\tau}$ associated to the problem $\left(S^{+}\right)$. In Section 3 we treat the nonexistence of radial positive solution for the Liouville problem $\left(S_{\infty}^{+}\right)$associated to $\left(S^{+}\right)$. This is the goal of Theorem 3.1. In Section 4 we get a priori estimates for the solutions of the system in the strongly coupled case and weakly coupled case. Finally in Section 5 we apply our results to obtain the proof of Theorems 1.1 and 1.2. 


\section{Notations}

Besides fixing notations, in this section we recall the results that we use throughout the paper. Let $R$ be a positive number; we consider the following space:

$$
\chi:=\left\{(u, v) \in C^{0}([0, R]) \times C^{0}([0, R]) \text { such that } u(R)=v(R)=0\right\}
$$

endowed with the norm $\|(u, v)\|=\|u\|_{\infty}+\|v\|_{\infty}$, which makes it a Banach space. Let $S_{\lambda}$ and $T_{\tau}: \chi \rightarrow \chi$ be the operators defined by $S_{\lambda}(u, v)=\left(S^{1}(u, v) ; S^{2}(u, v)\right)$ and $T_{\tau}(u, v)=\left(T^{1}(u, v) ; T^{2}(u, v)\right)$ such that

$$
\begin{aligned}
& S^{1}(u, v)(r):=\lambda^{\frac{1}{p-1}} \int_{r}^{R}\left[t^{1-N} \int_{0}^{t} s^{N-1}\left(a(s)|u(s)|^{\alpha}+b(s)|v(s)|^{\beta}\right) d s\right]^{\frac{1}{p-1}} d t, \\
& S^{2}(u, v)(r):=\lambda^{\frac{1}{q-1}} \int_{r}^{R}\left[t^{1-N} \int_{0}^{t} s^{N-1}\left(c(s)|u(s)|^{\gamma}+d(s)|v(s)|^{\delta}\right) d s\right]^{\frac{1}{q-1}} d t
\end{aligned}
$$

and

$$
\begin{aligned}
& T^{1}(u, v)(r):=\int_{r}^{R}\left[t^{1-N} \int_{0}^{t} s^{N-1}\left(a(s)|u(s)|^{\alpha}+b(s)|(v(s)+\tau)|^{\beta}\right) d s\right]^{\frac{1}{p-1}} d t, \\
& T^{2}(u, v)(r):=\int_{r}^{R}\left[t^{1-N} \int_{0}^{t} s^{N-1}\left(c(s)|u(s)|^{\gamma}+d(s)|v(s)|^{\delta}\right) d s\right]^{\frac{1}{q-1}} d t .
\end{aligned}
$$

It is well known that, for all $\lambda \in[0,1]$ and for all $\tau \in\left[0, \infty\left[, S_{\lambda}\right.\right.$ and $T_{\tau}$ are completely continuous operators on $\chi$. From the Maximum Principle this implies that $S_{\lambda}(\chi) \subset \chi$ and that the problem $\left(S^{+}\right)$is equivalent to find some non trivial positive fixed point $(u, v) \in \chi$ of the operator $\left(S_{1}\right)$ (by taking $\lambda=1$ ) such that $u^{\prime}(0)=v^{\prime}(0)=0$. The main difficulty will be to obtain suitable a priori estimates to guarantee that we are in the conditions of the fixed point theorem. To study this question, we use a Blow up argument like in Gidas-Spruck paper [9]. This technique transforms the problem $\left(S^{+}\right)$into a problem

$$
\left(S_{\infty}^{+}\right) \begin{cases}-\Delta_{p} u=A_{\infty}(\|y\|) u|u|^{\alpha-1}+B_{\infty}(\|y\|) v|v|^{\beta-1} & \text { in } \mathbf{R}^{N}, \\ -\Delta_{q} v=C_{\infty}(\|y\|) u|u|^{\gamma-1}+D_{\infty}(\|y\|) v|v|^{\delta-1} & \text { in } \mathbf{R}^{N} \\ u>0 \text { and } v>0 & \text { in } \mathbf{R}^{N}\end{cases}
$$

where $A_{\infty}, B_{\infty}, C_{\infty}$ and $D_{\infty}$ are positive continuous functions on $[0,+\infty[$. 
A Liouville type Theorem proves that $\left(S_{\infty}^{+}\right)$has no radial positive solutions. For the case $A_{\infty}=D_{\infty}=0, p=q=2, \beta>1$ and $\gamma>1$ this kind of theorems are obtained in [12] under the hypothesis

$$
\frac{1}{\beta+1}+\frac{1}{\gamma+1}>\frac{N-2}{2} \text {. }
$$

\section{Liouville's Theorem}

In this section we prove the nonexistence of radial positive solutions of the following Liouville's system $\left(S_{\infty}^{+}\right)$:

$$
\begin{array}{ll}
-\Delta_{p} u \geq a(x) u|u|^{\alpha-1}+b(x) v|v|^{\beta-1} & \text { in } \mathbf{R}^{N}, \\
-\Delta_{q} v \geq c(x) u|u|^{\gamma-1}+d(x) v|v|^{\delta-1} & \text { in } \mathbf{R}^{N} .
\end{array}
$$

We will need the following fundamental lemmas given in [5].

Lemma 3.1. Let $\left.\left.w \in C^{1}([0, R]) \cap C^{2}(] 0, R\right]\right), w \geq 0$, satisfying

$$
-\frac{d}{d r}\left(r^{N-1}\left|\frac{d w}{d r}(r)\right|^{m-2} \frac{d w}{d r}(r)\right) \geq 0 \quad \text { on } \quad[0, R],
$$

with $N>m>1$. Then, for any $r \in] 0, \frac{R}{2}[$ we have:

$$
w(r) \geq C_{N, m} r\left|\frac{d w}{d r}(r)\right|
$$

where

$$
C_{N, m}=\frac{m-1}{N-m}\left(1-2^{\frac{m-N}{m-1}}\right) .
$$

Lemma 3.2. Let be a positive function $w \in C^{0}\left(\left[0,+\infty[) \cap C^{2}(] 0,+\infty[)\right.\right.$ satisfying

$$
\left.-\frac{d}{d r}\left(r^{N-1}\left|\frac{d w}{d r}(r)\right|^{m-2} \frac{d w}{d r}(r)\right) \geq 0 \quad \text { in } \quad\right] 0,+\infty[
$$

with $N>m>1$. We suppose that for some real $r_{0} \geq 0$ we have $w\left(r_{0}\right)>0$. Then we obtain, $w(r)>0$ for all $r \geq r_{0}$ and there exists $C_{N, m}>0$ such that

$$
r^{\frac{N-m}{m-1}} w(r) \geq C_{N, m} \quad \text { for any } \quad r \geq r_{0} .
$$


Theorem 3.1. We assume $\left(H_{1}\right),\left(H_{3}\right),\left(H_{4}\right)$ and one of the following assumptions:

$\left(H_{s}\right) \max \left\{\frac{q \beta+p(q-1)}{\beta \gamma-(p-1)(q-1)}-\frac{N-p}{p-1} ; \frac{p \gamma+q(p-1)}{\beta \gamma-(p-1)(q-1)}-\frac{N-q}{q-1}\right\} \geq 0$

$\left(H_{w}\right) \quad \frac{N(p-1)}{N-p}>\alpha$ or $\frac{N(q-1)}{N-q}>\delta$.

Then the only radial positive solution of $\left(S_{\infty}^{+}\right)$is $(0,0)$.

Proof: We assume that the hypotheses $\left(H_{1}\right),\left(H_{3}\right)$ and $\left(H_{4}\right)$ hold:

1st case: If $\left(H_{s}\right)$ is satisfied, in [5] and [11] the authors prove the nonexistence of radial positive solutions of

$$
\begin{array}{ll}
-\Delta_{p} u \geq b(x) v|v|^{\beta-1} & \text { in } \mathbf{R}^{N}, \\
-\Delta_{q} v \geq c(x) u|u|^{\gamma-1} & \text { in } \mathbf{R}^{N} .
\end{array}
$$

Applying this result, we deduce the nonexistence of radial positive solutions of $\left(S_{\infty}^{+}\right)$.

2nd case: Let $u, v$ be a radial positive solution of the system $\left(S_{\infty}^{+}\right)$. We can rewrite it as

$$
\begin{aligned}
-\left(r^{N-1}\left|u^{\prime}(r)\right|^{p-2} u^{\prime}(r)\right)^{\prime} & =r^{N-1}\left[a(r)|u(r)|^{\alpha}+b(r)|v(r)|^{\beta}\right], \\
-\left(r^{N-1}\left|v^{\prime}(r)\right|^{q-2} v^{\prime}(r)\right)^{\prime} & =r^{N-1}\left[c(r)|u(r)|^{\gamma}+d(r)|v(r)|^{\delta}\right] \\
u^{\prime}(0) & =v^{\prime}(0)=0 .
\end{aligned}
$$

Integrating (3.10) and (3.11) on $(0, r)$ and taking into account that $u^{\prime}(r)$, $v^{\prime}(r)<0$, for $r>0$, we find

$$
\begin{aligned}
& -u^{\prime}(r) \geq\left(\frac{a(0)}{N}\right)^{\frac{1}{p-1}} r^{\frac{1}{p-1}}(u(r))^{\frac{\alpha}{p-1}} \\
& -v^{\prime}(r) \geq\left(\frac{d(0)}{N}\right)^{\frac{1}{q-1}} r^{\frac{1}{q-1}}(v(r))^{\frac{\delta}{q-1}} .
\end{aligned}
$$

Thus, from Lemma 3.1 we deduce that there exists some constant $C>0$ depending only of $(N, p, q, a, d)$ such that

$$
\begin{aligned}
& u(r) \geq-C_{N, p} r u^{\prime}(r) \geq C r^{\frac{p}{p-1}}(u(r))^{\frac{\alpha}{p-1}} \\
& v(r) \geq-C_{N, q} r v^{\prime}(r) \geq C r^{\frac{q}{q-1}}(v(r))^{\frac{\delta}{q-1}}
\end{aligned}
$$

for all $r \in] 0,+\infty[$. 
Thus, by Lemma 3.2, there exists some constant $C>0$ depending only of $(N, p, q, a, d)$ and $r_{0}>0$ such that for all $r \geq r_{0}$, we have

$$
1 \geq C r^{\frac{p}{p-1}-\frac{N-p}{p-1} \frac{\alpha-p+1}{p-1}}
$$

and

$$
1 \geq C r^{\frac{q}{q-1}-\frac{N-q}{q-1} \frac{\delta-q+1}{q-1}} .
$$

Consequently if $\left(H_{w}\right)$ is satisfied, we get

$$
\frac{p}{p-1}-\frac{N-p}{p-1} \frac{\alpha-p+1}{p-1}>0,
$$

or

$$
\frac{q}{q-1}-\frac{N-q}{q-1} \frac{\delta-q+1}{q-1}>0
$$

Hence a contradiction.

\section{A priori bounds for positive solutions of $\left(S^{+}\right)$}

In this section we will study a priori bounds for radial positive solutions of the system $\left(S^{+}\right)$, in the strongly coupled and weakly coupled cases. For that, we need the following lemma:

Lemma 4.1. We assume that there is a sequence $\left\{\left(\tilde{u}_{n}, \tilde{v}_{n}\right)\right\}$ in $\left.\left.\left(C^{1}\left(\left[0 . R_{n}\right]\right) \cap C^{2}(] 0, R_{n}\right]\right)\right)^{2}$ of positive solutions of the following system $\left(\tilde{S}_{n}\right)$ :

$$
\begin{gathered}
-\frac{d}{d y}\left(y^{N-1}\left|\frac{d \tilde{u}_{n}}{d y}(y)\right|^{p-2} \frac{d \tilde{u}_{n}}{d y}(y)\right)=y^{N-1} F_{n}\left(\tilde{u}_{n}(y), \tilde{v}_{n}(y)\right) \\
-\frac{d}{d y}\left(y^{N-1}\left|\frac{d \tilde{v}_{n}}{d y}(y)\right|^{q-2} \frac{d \tilde{v}_{n}}{d y}(y)\right)=y^{N-1} G_{n}\left(\tilde{u}_{n}(y), \tilde{v}_{n}(y)\right), \\
\frac{d \tilde{u}_{n}}{d y}(0)=\frac{d \tilde{v}_{n}}{d y}(0)=\tilde{u}_{n}\left(R_{n}\right)=\tilde{v}_{n}\left(R_{n}\right)=0
\end{gathered}
$$

where

$$
\left\{\begin{array}{l}
F_{n}\left(\tilde{u}_{n}(y), \tilde{v}_{n}(y)\right)=\left[A_{n}(|y|)\left|\tilde{u}_{n}(y)\right|^{\alpha}+B_{n}(|y|)\left|\tilde{v}_{n}(y)+\tau_{n}^{\prime}\right|^{\beta}\right], \\
G_{n}\left(\tilde{u}_{n}(y), \tilde{v}_{n}(y)\right)=\left[C_{n}(|y|)\left|\tilde{u}_{n}(y)\right|^{\gamma}+D_{n}(|y|)\left|\tilde{v}_{n}(y)\right|^{\delta}\right],
\end{array}\right.
$$


where $\left\{\left(A_{n}, B_{n}, C_{n}, D_{n}\right)\right\}$ are sequences of positive functions in $\left(C_{\mathrm{loc}}^{0}\left([0,+\infty[))^{4}\right.\right.$ which converge to $\left(A_{\infty}, B_{\infty}, C_{\infty}, D_{\infty}\right)$ in $\left(C_{\mathrm{loc}}^{0}\left([0,+\infty[))^{4}\right.\right.$. If,

$$
\lim _{n \rightarrow \infty} \tau_{n}^{\prime}=0, \quad \lim _{n \rightarrow \infty} R_{n}=+\infty,
$$

and $0<\left\|\left(\tilde{u}_{n}(0), \tilde{v}_{n}(0)\right)\right\| \leq 1$ for all $n \in \mathbf{N}$, then there exists a subsequence $\left\{\left(\tilde{u}_{n_{k}}, \tilde{v}_{n_{k}}\right)\right\}$ of $\left\{\left(\tilde{u}_{n}, \tilde{v}_{n}\right)\right\}$ converging in $\left(C_{\mathrm{loc}}^{0}\left([0,+\infty[))^{2}\right.\right.$ and whose limit $(\tilde{u}, \tilde{v})$ is a positive radially symmetric solution of the following Liouville's system

$$
\left(S_{\infty}^{+}\right)\left\{\begin{aligned}
-\Delta_{p} u & =A_{\infty}(|y|) u|u|^{\alpha-1}+B_{\infty}(|y|) v|v|^{\beta-1} & & \text { in } \mathbf{R}^{N} \\
-\Delta_{q} v & =C_{\infty}(|y|) u|u|^{\gamma-1}+D_{\infty}(|y|) v|v|^{\delta-1} & & \text { in } \mathbf{R}^{N} .
\end{aligned}\right.
$$

Proof: We argue as in [5]. We first note that, since $\left\|\left(\tilde{u}_{n}(0), \tilde{v}_{n}(0)\right)\right\| \leq 1$ there exists a subsequence $\left\{\left(\tilde{u}_{n_{k}}(0), \tilde{v}_{n_{k}}(0)\right)\right\}$ which converges in $\mathbf{R}^{2}$ to $\left(\tilde{u}_{0}, \tilde{v}_{0}\right)$ such that $\left\|\left(\tilde{u}_{0}, \tilde{v}_{0}\right)\right\| \leq 1$.

Next we will prove that the restriction of $\left\{\left(\tilde{u}_{n}, \tilde{v}_{n}\right)\right\}$ to $\left[0, R^{\prime}\right]$ is equicontinuous in $\left(C^{0}\left(\left[0, R^{\prime}\right]\right)\right)^{2}$.

In fact, multiplying (4.1) by $\frac{d \tilde{u}_{n}}{d y}$ and (4.2) by $\frac{d \tilde{v}_{n}}{d y}$ we obtain the following equations:

$$
\begin{aligned}
\frac{p-1}{p} \frac{d}{d y}\left(\left|\frac{d \tilde{u}_{n}}{d y}(y)\right|^{p}\right) & +\frac{N-1}{y}\left|\frac{d \tilde{u}_{n}}{d y}(y)\right|^{p} \\
& +F_{n}\left(\tilde{u}_{n}(y), \tilde{v}_{n}(y)\right) \frac{d \tilde{u}_{n}}{d y}(y)=0 \\
\frac{q-1}{q} \frac{d}{d y}\left(\left|\frac{d \tilde{v}_{n}}{d y}(y)\right|^{q}\right) & +\frac{N-1}{y}\left|\frac{d \tilde{v}_{n}}{d y}(y)\right|^{q} \\
& +G_{n}\left(\tilde{u}_{n}(y), \tilde{v}_{n}(y)\right) \frac{d \tilde{v}_{n}}{d y}(y)=0 .
\end{aligned}
$$

From (4.5) and (4.6) it follows that

$$
\begin{aligned}
& \frac{p-1}{p} \frac{d}{d y}\left(\left|\frac{d \tilde{u}_{n}}{d y}(y)\right|^{p}\right)+a_{n} \frac{d \tilde{u}_{n}}{d y}(y) \leq 0 \\
& \frac{q-1}{q} \frac{d}{d y}\left(\left|\frac{d \tilde{v}_{n}}{d y}(y)\right|^{q}\right)+b_{n} \frac{d \tilde{v}_{n}}{d y}(y) \leq 0
\end{aligned}
$$

where

$$
a_{n}=2 \max _{s \in\left[0, R^{\prime}\right]}\left\{A_{n} ; B_{n}\right\} \quad \text { and } \quad b_{n}=2 \max _{s \in\left[0, R^{\prime}\right]}\left\{C_{n} ; D_{n}\right\}
$$


Since the sequence $\left\{\left(A_{n}, B_{n}, C_{n}, D_{n}\right)\right\}$ converges to $\left(A_{\infty}, B_{\infty}, C_{\infty}, D_{\infty}\right)$ in $\left(C^{0}\left(\left[0, R^{\prime}\right]\right)\right)^{4}$, then there exist $a_{1}>0$ and $b_{1}>0$ such that $\left\|a_{n}\right\|<a_{1}$ and $\left\|b_{n}\right\|<b_{1}$ for all $n \in \mathbf{N}$. Hence, from (4.7), (4.8) and by integrating from 0 to $y \in\left[0, R^{\prime}\right]$ we find that

$$
\begin{aligned}
& \frac{p-1}{p}\left|\frac{d \tilde{u}_{n}}{d y}(y)\right|^{p}+a_{1} \int_{0}^{y} \frac{d \tilde{u}_{n}}{d y}(s) d s \leq 0 \\
& \frac{q-1}{q}\left|\frac{d \tilde{v}_{n}}{d y}(y)\right|^{q}+b_{1} \int_{0}^{y} \frac{d \tilde{v}_{n}}{d y}(s) d s \leq 0
\end{aligned}
$$

and hence that

$$
\begin{aligned}
& \left|\frac{d \tilde{u}_{n}}{d y}(y)\right| \leq C_{1} \\
& \left|\frac{d \tilde{v}_{n}}{d y}(y)\right| \leq C_{2}
\end{aligned}
$$

uniformly in $n$, which imply the equicontinuity of the sequence $\left\{\left(\tilde{u}_{n}, \tilde{v}_{n}\right)\right\}$. Thus, by the Ascoli-Arzelà Theorem, there exists a subsequence $\left\{\left(\tilde{u}_{n_{k}}, \tilde{v}_{n_{k}}\right)\right\}$, such that $\left\{\left(\tilde{u}_{n_{k}}, \tilde{v}_{n_{k}}\right)\right\}$ converges to $(\tilde{u}, \tilde{v})$ when $k \rightarrow \infty$, in $\left(C^{0}\left(\left[0, R^{\prime}\right]\right)\right)^{2}$. Now from (4.1) and (4.2) we have that $\left\{\left(\tilde{u}_{n_{k}}, \tilde{v}_{n_{k}}\right)\right\}$ satisfy

(4.14) $\tilde{u}_{n_{k}}(0)-\tilde{u}_{n_{k}}(y)=\int_{0}^{y}\left(\frac{1}{t^{N-1}} \int_{0}^{t} s^{N-1} F_{n_{k}}\left(\tilde{u}_{n_{k}}(s), \tilde{v}_{n_{k}}(s)\right) d s\right)^{\frac{1}{p-1}} d t$

(4.15) $\tilde{v}_{n_{k}}(0)-\tilde{v}_{n_{k}}(y)=\int_{0}^{y}\left(\frac{1}{t^{N-1}} \int_{0}^{t} s^{N-1} G_{n_{k}}\left(\tilde{u}_{n_{k}}(s), \tilde{v}_{n_{k}}(s)\right) d s\right)^{\frac{1}{q-1}} d t$.

By the Dominated Convergence Theorem we obtain that $(\tilde{u}, \tilde{v})$ satisfy

$$
\begin{aligned}
& \tilde{u_{0}}-\tilde{u}(y)=\int_{0}^{y}\left(\frac{1}{t^{N-1}} \int_{0}^{t} s^{N-1} F_{\infty}(\tilde{u}(s), \tilde{v}(s)) d s\right)^{\frac{1}{p-1}} d t \\
& \tilde{v_{0}}-\tilde{v}(y)=\int_{0}^{y}\left(\frac{1}{t^{N-1}} \int_{0}^{t} s^{N-1} G_{\infty}(\tilde{u}(s), \tilde{v}(s)) d s\right)^{\frac{1}{q-1}} d t
\end{aligned}
$$

where

$$
\begin{aligned}
F_{\infty}(\tilde{u}(s), \tilde{v}(s)) & =\left[A_{\infty}(s)|\tilde{u}(s)|^{\alpha}+B_{\infty}(s)|\tilde{v}(s)|^{\beta}\right], \\
G_{\infty}(\tilde{u}(s), \tilde{v}(s)) & =\left[C_{\infty}(s)|\tilde{u}(s)|^{\gamma}+D_{\infty}(s)|\tilde{v}(s)|^{\delta}\right],
\end{aligned}
$$


which imply that $\tilde{u} \geq 0$ and $\tilde{v} \geq 0$ belong to $\left.\left.\left(C^{1}\left(\left[0, R^{\prime}\right]\right) \cap C^{2}(] 0, R^{\prime}\right]\right)\right)^{2}$ and satisfy

$$
\begin{aligned}
& -\frac{d}{d y}\left(y^{N-1}\left|\frac{d \tilde{u}}{d y}(y)\right|^{p-2} \frac{d \tilde{u}}{d y}(y)\right)=y^{N-1} F_{\infty}(\tilde{u}(y), \tilde{v}(y)), \\
& -\frac{d}{d y}\left(y^{N-1}\left|\frac{d \tilde{v}}{d y}(y)\right|^{q-2} \frac{d \tilde{v}}{d y}(y)\right)=y^{N-1} G_{\infty}(\tilde{u}(y), \tilde{v}(y)),
\end{aligned}
$$

for all $y \in\left[0, R^{\prime}\right]$ and

$$
\frac{d \tilde{u}}{d y}(0)=\frac{d \tilde{v}}{d y}(0)=0 .
$$

We claim that $\tilde{u}$ and $\tilde{v}$ can be extended to $[0,+\infty[$. It is sufficient to note that we can repeat the above argument on an interval $[0, \bar{R}], \bar{R}>R^{\prime}$, for the convergent sequence $\left\{\left(\tilde{u}_{n_{k}}, \tilde{v}_{n_{k}}\right)\right\}$ on $\left[0, R^{\prime}\right]$. We obtain in this manner $(\bar{u}, \bar{v})$ as solution of $(4.18),(4.19)$ and $(4.20)$ on $[0, \bar{R}]$ and which $\bar{u}, \bar{v}$ satisfy

$$
\bar{u}(y)=\tilde{u}(y) \quad \text { and } \quad \bar{v}(y)=\tilde{v}(y) \quad \text { on } \quad[0, \bar{R}]
$$

It is now clear that $\bar{u}, \bar{v}$ can be extended to $[0,+\infty$ [ as a solution of $\left(S_{\infty}^{+}\right)$which is such that $\bar{u} \geq 0, \bar{v} \geq 0$ for all $y \in[0, \infty[$ and Lemma 4.1 follows.

\subsection{The strongly coupled case.}

Proposition 4.1. We assume that the system $\left(S^{+}\right)$is strongly coupled.

Under the assumptions $\left(H_{1}\right),\left(H_{2}\right),\left(H_{3}\right),\left(H_{4}\right)$ and $\left(H_{s}\right)$, there exists some $C_{0}>0$ such that for all $\tau \in[0, \infty[$ and for all fixed points $(u, v) \in \chi$ of $T_{\tau}$ we have

$$
\|(u, v)\|<C_{0} .
$$

Proof: We suppose that there exist sequences $\left\{\left(\tau_{n}\right)\right\}$ and $\left\{\left(u_{n}, v_{n}\right)\right\}$ satisfying

$$
\left(u_{n}, v_{n}\right)=T_{\tau_{n}}\left(u_{n}, v_{n}\right) \quad \forall n \in \mathbf{N}
$$

and such that, at least for a subsequence; $\left\|\left(u_{n}, v_{n}\right)\right\| \rightarrow+\infty$ as $n \rightarrow+\infty$. 
From the Maximum Principle (4.23) is equivalent that $\left(u_{n}, v_{n}\right)$ satisfies in $[0, R]$

$$
\begin{aligned}
-\left(r^{N-1}\left|u_{n}^{\prime}(r)\right|^{p-2} u_{n}^{\prime}(r)\right)^{\prime}= & r^{N-1}\left[a(r)\left|u_{n}(r)\right|^{\alpha}\right. \\
& \left.+b(r)\left|v_{n}(r)+\tau_{n}\right|^{\beta}\right], \\
-\left(r^{N-1}\left|v_{n}^{\prime}(r)\right|^{q-2} v_{n}^{\prime}(r)\right)^{\prime}= & r^{N-1}\left[c(r)\left|u_{n}(r)\right|^{\gamma}\right. \\
& \left.+d(r)\left|v_{n}(r)\right|^{\delta}\right], \\
u_{n}^{\prime}(0)=v_{n}^{\prime}(0)=u_{n}(R)= & v_{n}(R)=0 .
\end{aligned}
$$

We introduce new functions $\tilde{u}_{n}$ and $\tilde{v}_{n}$ in the following way:

$$
\begin{aligned}
& \tilde{u}_{n}(y)=\frac{u_{n}(r)}{\sigma_{n}{ }^{l}}, \\
& \tilde{v}_{n}(y)=\frac{v_{n}(r)}{\sigma_{n}{ }^{k}},
\end{aligned}
$$

and we make the change of variables

$$
y=\sigma_{n} r \quad \text { on } \quad[0, R],
$$

where

$$
\sigma_{n}=\left(u_{n}(0)\right)^{\frac{1}{l}}+\left(v_{n}(0)\right)^{\frac{1}{k}}
$$

and $l, k$ are positive numbers to be chosen below.

In this way we obtain that $\tilde{u}_{n}$ and $\tilde{v}_{n}$ are defined on the interval $\left[0, R \sigma_{n}\right]$ and satisfy the following system $\left(\tilde{S}_{n}^{+}\right)$

$$
\begin{gathered}
-\frac{d}{d y}\left(y^{N-1}\left|\frac{d \tilde{u}_{n}}{d y}(y)\right|^{p-2} \frac{d \tilde{u}_{n}}{d y}(y)\right)=y^{N-1} F_{n}\left(\tilde{u}_{n}(y), \tilde{v}_{n}(y)\right), \\
-\frac{d}{d y}\left(y^{N-1}\left|\frac{d \tilde{v}_{n}}{d y}(y)\right|^{q-2} \frac{d \tilde{v}_{n}}{d y}(y)\right)=y^{N-1} G_{n}\left(\tilde{u}_{n}(y), \tilde{v}_{n}(y)\right), \\
\frac{d \tilde{u}_{n}}{d y}(0)=\frac{d \tilde{v}_{n}}{d y}(0)=\tilde{u}_{n}\left(R_{n}\right)=\tilde{v}_{n}\left(R_{n}\right)=0
\end{gathered}
$$

where

$$
\begin{aligned}
F_{n}\left(\tilde{u}_{n}(y), \tilde{v}_{n}(y)\right) & =\left[A_{n}(y)\left|\tilde{u}_{n}(y)\right|^{\alpha}+B_{n}(y)\left|\tilde{v}_{n}(y)+\frac{\tau_{n}}{\sigma_{n}^{k}}\right|^{\beta}\right], \\
G_{n}\left(\tilde{u}_{n}(y), \tilde{v}_{n}(y)\right) & =\left[C_{n}(y)\left|\tilde{u}_{n}(y)\right|^{\gamma}+D_{n}(y)\left|\tilde{v}_{n}(y)\right|^{\delta}\right]
\end{aligned}
$$


and

(4.34) $A_{n}(y)=a\left(\frac{y}{\sigma_{n}}\right) \sigma_{n}^{-p+l(\alpha-p+1)} B_{n}(y)=b\left(\frac{y}{\sigma_{n}}\right) \sigma_{n}^{-p-l(p-1)+k \beta}$,

$C_{n}(y)=c\left(\frac{y}{\sigma_{n}}\right) \sigma_{n}^{-q+k(q-1)+l \gamma} D_{n}(y)=d\left(\frac{y}{\sigma_{n}}\right) \sigma_{n}^{-q+k(\delta-q+1)}$

$$
R_{n}=R \sigma_{n}
$$

By choosing

$$
l=\frac{p(q-1)+\beta q}{\beta \gamma-(p-1)(q-1)} \quad \text { and } \quad k=\frac{q(p-1)+p \gamma}{\beta \gamma-(p-1)(q-1)},
$$

we obtain

$$
\begin{array}{ll}
A_{n}(y)=a\left(\frac{y}{\sigma_{n}}\right) \sigma_{n}^{l \alpha-k \beta}, & B_{n}(y)=b\left(\frac{y}{\sigma_{n}}\right) \\
C_{n}(y)=c\left(\frac{y}{\sigma_{n}}\right), & D_{n}(y)=d\left(\frac{y}{\sigma_{n}}\right) \sigma_{n}^{k \delta-l \gamma} .
\end{array}
$$

Recall that $\tilde{u}_{n}, \tilde{v}_{n}$ satisfy

$$
\begin{array}{lll}
\frac{d \tilde{u}_{n}}{d y}(y) \leq 0, & \tilde{u}_{n}(y) \leq 1 & \forall y \in\left[0, R_{n}\right], \\
\frac{d \tilde{v}_{n}}{d y}(y) \leq 0, & \tilde{v}_{n}(y) \leq 1 & \forall y \in\left[0, R_{n}\right]
\end{array}
$$

and

$$
\left(\tilde{u}_{n}(0)\right)^{\frac{1}{l}}+\left(\tilde{v}_{n}(0)\right)^{\frac{1}{k}}=1 .
$$

Thus in order to apply Lemma 4.1 , it remains to estimate $\tau_{n}^{\prime}=\frac{\tau_{n}}{\sigma_{n}^{k}}$.

By integrating (4.24) from 0 to $r \in[0, R]$

$$
-u_{n}{ }^{\prime}(r) \geq C r^{\frac{1}{p-1}}\left(v_{n}(r)+\tau_{n}\right)^{\frac{\beta}{p-1}},
$$

thus from (4.43)

$$
-u_{n}^{\prime}(r) \geq C r^{\frac{1}{p-1}} \tau_{n}^{\frac{\beta}{p-1}}
$$

integrating this inequality from 0 to $R$, we obtain that

$$
u_{n}(0) \geq C R^{\frac{p}{p-1}} \tau_{n}^{\frac{\beta}{p-1}} .
$$


Then, from (4.45), we have

$$
\tau_{n} \leq C\left(u_{n}(0)\right)^{\frac{p-1}{\beta}}
$$

and hence,

$$
\frac{\tau_{n}}{\sigma_{n}^{k}} \leq C \frac{\left(u_{n}(0)\right)^{\frac{p-1}{\beta}}}{\sigma_{n}^{k}} .
$$

By definition of $\sigma_{n}, 0 \leq u_{n}(0) \leq \sigma_{n}^{l}$. Hence,

$$
\frac{\tau_{n}}{\sigma_{n}^{k}} \leq C \sigma_{n}^{\frac{l(p-1)-k \beta}{\beta}} .
$$

Thus, by the definition (4.37) of $l$ and $k$, we have

$$
l(p-1)-k \beta=-p \text {. }
$$

Then

$$
\frac{\tau_{n}}{\sigma_{n}^{k}} \leq C \sigma_{n}^{\frac{-p}{\beta}}
$$

and hence $\tau_{n}^{\prime}=\frac{\tau_{n}}{\sigma_{n}^{k}} \rightarrow 0$ as $n \rightarrow+\infty$. Moreover, we have that $\left\{\left(A_{n}, B_{n}, C_{n}, D_{n}\right)\right\}$ converges to $\left(A_{\infty}, b(0), c(0), D_{\infty}\right)$ in $C_{\text {loc }}^{0}([0,+\infty[)$, where $A_{\infty}=0$ or $a(0)$ and $D_{\infty}=0$ or $d(0)$. Consequently, in the sense of Lemma 4.1, the sequence $\left\{\left(\tilde{u}_{n}, \tilde{v}_{n}\right)\right\}$ converges to $(\tilde{u}, \tilde{v})$, such that $(\tilde{u}, \tilde{v})$ satisfies

$$
\left(S_{\infty}\right) \begin{cases}-\Delta_{p} \tilde{u}=A_{\infty} \tilde{u}|\tilde{u}|^{\alpha-1}+b(0) \tilde{v}|\tilde{v}|^{\beta-1} & \text { in } \mathbf{R}^{N}, \\ -\Delta_{q} \tilde{v}=c(0) \tilde{u}|\tilde{u}|^{\gamma-1}+D_{\infty} \tilde{v}|\tilde{v}|^{\delta-1} & \text { in } \mathbf{R}^{N} .\end{cases}
$$

Thus, from Lemma 3.2, we have $\tilde{u}>0$ and $\tilde{v}>0$. But, since $\left(H_{s}\right)$ is satisfied then, from Liouville's Theorem 3.1, the problem $\left(S_{\infty}\right)$ has no radial positive solution. Hence we obtain a contradiction and Proposition 4.1 is proved.

\subsection{The weakly coupled case.}

Before studying this case, we make some remarks on the sequence $\left\{\left(\tilde{u}_{n}, \tilde{v}_{n}\right)\right\}$ defined in the proof of Proposition 4.1, that is:

Claim 1. If

$$
\lim _{n \rightarrow+\infty} \tilde{u}_{n}(0)=0
$$

then we have

$$
\lim _{n \rightarrow+\infty} \tilde{v}_{n}(0)>0 \quad \text { and } \quad \gamma<\frac{p \gamma+q(p-1)}{q \beta+p(q-1)} \delta .
$$


Proof: Before proving that, we will denote by $C$ any positive constant depending only on the data. Now, let $t_{n}$ be such that $\tilde{v}_{n}\left(t_{n}\right)=\frac{\tilde{v}_{n}(0)}{2}$. Since $\tilde{u}_{n}^{\prime} \leq 0$ and $\tilde{v}_{n}^{\prime} \leq 0$, by integrating the equation (4.32) on $[0, y]$ and later on $\left[0, t_{n}\right]$, we obtain

$$
0<\frac{\tilde{v}_{n}(0)}{2}=\tilde{v}_{n}(0)-\tilde{v}_{n}\left(t_{n}\right) \leq C\left[t_{n}^{q}\left|\tilde{u}_{n}(0)\right|^{\gamma}+t_{n}^{q} \sigma_{n}^{k \delta-l \gamma}\left|\tilde{v}_{n}(0)\right|^{\delta}\right]^{\frac{1}{q-1}}
$$

Moreover, by integrating (4.31) and (4.32) from 0 to $t_{n}$, we get

$$
\begin{aligned}
& -\tilde{u}_{n}^{\prime}\left(t_{n}\right) \geq C t_{n}^{\frac{1}{p-1}}\left(\tilde{v}_{n}\left(t_{n}\right)\right)^{\frac{\beta}{p-1}}, \\
& -\tilde{v}_{n}^{\prime}\left(t_{n}\right) \geq C t_{n}^{\frac{1}{q-1}} \sigma_{n}^{k \delta-l \gamma}\left(\tilde{v}_{n}\left(t_{n}\right)\right)^{\frac{\delta}{q-1}} .
\end{aligned}
$$

On the other hand, from Lemma 3.1 and (4.50)-(4.51) we have

$$
\left|\tilde{u}_{n}(0)\right|^{p-1} \geq\left|\tilde{u}_{n}\left(t_{n}\right)\right|^{p-1} \geq C t_{n}^{p}\left|\tilde{v}_{n}\left(t_{n}\right)\right|^{\beta} \geq C_{N, p} t_{n}^{p}\left|\frac{\tilde{v}_{n}(0)}{2}\right|^{\beta}
$$

and

$$
\left|\tilde{v}_{n}(0)\right|^{q-1} \geq\left|\tilde{v}_{n}\left(t_{n}\right)\right|^{q-1} \geq C t_{n}^{q} \sigma_{n}^{k \delta-l \gamma}\left|\tilde{v}_{n}(0)\right|^{\delta} .
$$

Then, since $\left(\tilde{u}_{n}(0)\right)^{l}+\left(\tilde{v}_{n}(0)\right)^{k}=1$ and

$$
\lim _{n \rightarrow+\infty} \tilde{u}_{n}(0)=0
$$

we obtain

$$
\lim _{n \rightarrow+\infty} \tilde{v}_{n}(0)>0 .
$$

Thus, from (4.52), (4.54) and (4.55) we have

$$
\lim _{n \rightarrow+\infty} t_{n}=0 \text {. }
$$

Hence, from (4.49), (4.55), (4.56) and (4.57) we deduce that

$$
0<\lim \sup t_{n}^{q} \sigma_{n}^{k \delta-l \gamma}<+\infty .
$$

Consequently, from (4.56), (4.57) and since we assume that

$$
\lim _{n \rightarrow+\infty} \sigma_{n}=+\infty
$$

then we have $k \delta-l \gamma>0$. By definition of numbers $k$, $l$ Claim 1 follows.

Proposition 4.2. We assume that the system $\left(S^{+}\right)$is weakly coupled.

Under the assumptions $\left(H_{1}\right),\left(H_{2}\right),\left(H_{3}\right),\left(H_{4}\right)$, and $\left(H_{w}\right)$, there exists some $C_{0}>0$ such that for all $\tau \in[0, \infty[$ and for all fixed points $(u, v) \in \chi$ of $T_{\tau}$ we have

$$
\|(u, v)\|<C_{0} .
$$


Proof: We assume $\left(H_{1}\right),\left(H_{2}\right),\left(H_{3}\right),\left(H_{4}\right)$, and $\left(H_{w}\right)$. Suppose that

$$
\text { j) } \beta<\frac{q \beta+p(q-1)}{p \gamma+q(p-1)} \alpha
$$

is satisfied. Then as in the proof of Proposition 4.1, we suppose that there exist the sequences $\left\{\left(\tau_{n}\right)\right\}$ and $\left\{\left(u_{n}, v_{n}\right)\right\}$ which, at least for a subsequence, $\left\|\left(u_{n}, v_{n}\right)\right\| \rightarrow+\infty$ as $n \rightarrow+\infty$, satisfy $\left(u_{n}, v_{n}\right)=T_{\tau_{n}}\left(u_{n}, v_{n}\right)$. It is equivalent that $\left(u_{n}, v_{n}\right)$ satisfies in $[0, R]$

$$
\begin{aligned}
-\left(r^{N-1}\left|u_{n}^{\prime}(r)\right|^{p-2} u_{n}^{\prime}(r)\right)^{\prime}= & r^{N-1}\left[a(r)\left|u_{n}(r)\right|^{\alpha}\right. \\
& \left.+b(r)\left|v_{n}(r)+\tau_{n}\right|^{\beta}\right], \\
-\left(r^{N-1}\left|v_{n}^{\prime}(r)\right|^{q-2} v_{n}^{\prime}(r)\right)^{\prime}= & r^{N-1}\left[c(r)\left|u_{n}(r)\right|^{\gamma}\right. \\
& \left.+d(r)\left|v_{n}(r)\right|^{\delta}\right], \\
u_{n}^{\prime}(0)=v_{n}^{\prime}(0)=u_{n}(R)= & v_{n}(R)=0 .
\end{aligned}
$$

We introduce new functions $\tilde{u}_{n}$ and $\tilde{v}_{n}$ in the following way:

$$
\begin{aligned}
& \bar{u}_{n}(y)=\frac{u_{n}(r)}{\sigma_{n}^{l}}, \\
& \bar{v}_{n}(y)=\frac{v_{n}(r)}{\sigma_{n}{ }^{k}} .
\end{aligned}
$$

We make the change of variables

$$
y=\frac{\sigma_{n}}{t_{n}} r \quad \text { on } \quad[0, R]
$$

where

$$
\sigma_{n}=\left(u_{n}(0)\right)^{\frac{1}{l}}+\left(v_{n}(0)\right)^{\frac{1}{k}}
$$

and where $\left(t_{n}\right)_{n \in N}$ is some sequence to be chosen below.

In this way we obtain the following equations for $\bar{u}_{n}$ and $\bar{v}_{n}$ defined on the interval $\left[0, \frac{\sigma_{n}}{t_{n}} R\right]$ and

$$
\left\{\begin{array}{l}
-\frac{d}{d y}\left(y^{N-1}\left|\frac{d \bar{u}_{n}}{d y}(y)\right|^{p-2} \frac{d \bar{u}_{n}}{d y}(y)\right)=y^{N-1} F_{n}\left(\bar{u}_{n}(y), \bar{v}_{n}(y)\right), \\
-\frac{d}{d y}\left(y^{N-1}\left|\frac{d \bar{v}_{n}}{d y}(y)\right|^{q-2} \frac{d \bar{v}_{n}}{d y}(y)\right)=y^{N-1} G_{n}\left(\bar{u}_{n}(y), \bar{v}_{n}(y)\right), \\
\frac{d \bar{u}_{n}}{d y}(0)=\frac{d \bar{v}_{n}}{d y}(0)=\bar{u}_{n}\left(R_{n}\right)=\bar{v}_{n}\left(R_{n}\right)=0
\end{array}\right.
$$


where

$(4.65)$

$$
F_{n}\left(\bar{u}_{n}(y), \bar{v}_{n}(y)\right)=\left[A_{n}(y)\left|\bar{u}_{n}(y)\right|^{\alpha}+B_{n}(y)\left|\bar{v}_{n}(y)+\frac{\tau_{n}}{\sigma_{n}^{k}}\right|^{\beta}\right],
$$

$$
G_{n}\left(\bar{u}_{n}(y), \bar{v}_{n}(y)\right)=\left[C_{n}(y)\left|\bar{u}_{n}(y)\right|^{\gamma}+D_{n}(y)\left|\bar{v}_{n}(y)\right|^{\delta}\right]
$$

and

$$
\begin{aligned}
& A_{n}(y)=a\left(\frac{y t_{n}}{\sigma_{n}}\right) t_{n}^{p} \sigma_{n}^{-p+l(\alpha-p+1)} \\
& B_{n}(y)=b\left(\frac{y t_{n}}{\sigma_{n}}\right) t_{n}^{p} \sigma_{n}^{-p-l(p-1)+k \beta}, \\
& C_{n}(y)=c\left(\frac{y t_{n}}{\sigma_{n}}\right) t_{n}^{q} \sigma_{n}^{-q+k(q-1)+l \gamma} \\
& D_{n}(y)=d\left(\frac{y t_{n}}{\sigma_{n}}\right) t_{n}^{q} \sigma_{n}^{-q+k(\delta-q+1)},
\end{aligned}
$$

$$
R_{n}=\frac{\sigma_{n}}{t_{n}} R
$$

By choosing the positive numbers $l$ and $k$ as in (4.37) we obtain

$$
\begin{array}{ll}
A_{n}(y)=a\left(\frac{y t_{n}}{\sigma_{n}}\right) t_{n}^{p} \sigma_{n}^{l \alpha-k \beta}, & B_{n}(y)=b\left(\frac{y t_{n}}{\sigma_{n}}\right) t_{n}^{p}, \\
C_{n}(y)=c\left(\frac{y t_{n}}{\sigma_{n}}\right) t_{n}^{q}, & D_{n}(y)=d\left(\frac{y t_{n}}{\sigma_{n}}\right) t_{n}^{q} \sigma_{n}{ }^{k \delta-l \gamma} .
\end{array}
$$

1st case: $\lim _{n \rightarrow+\infty} \bar{u}_{n}(0)>0$.

By choosing $t_{n}=\sigma_{n}^{\frac{-l \alpha+k \beta}{p}}$, as in Lemma 4.1, we obtain that the first equation of the system $\left(S_{n}^{+}\right)$converges to the equation

$$
-\Delta_{p} \bar{u}=a(0) \bar{u}|\bar{u}|^{\beta-1} \quad \text { in } \quad R^{N},
$$

where $\bar{u}>0$ is the limit of $\bar{u}_{n}$.

Thus, from $\left(H_{w}\right)$ and Liouville's Theorem 3.1, the equation (4.72) has no radial positive solution. Hence the contradiction follows. 
2nd case: $\lim _{n \rightarrow+\infty} \bar{u}_{n}(0)=0$.

We recall that $\bar{u}_{n}(0)=\tilde{u}_{n}(0)$ and $\bar{v}_{n}(0)=\tilde{v}_{n}(0)$ where $\left(\tilde{u}_{n}, \tilde{v}_{n}\right)$ are the functions defined in the proof of Proposition 4.2. Then from our claim, we deduce that

$$
\lim _{n \rightarrow+\infty} \bar{v}_{n}(0)>0 \quad \text { and } \quad \gamma<\frac{p \gamma+q(p-1)}{q \beta+p(q-1)} \delta .
$$

Consequently, in this case it suffices to choose $t_{n}=\sigma_{n}^{\frac{-k \delta+l \gamma}{q}}$. Then, we obtain that $\left\{\bar{v}_{n}\right\}$ converges to $\bar{v}$ and $\bar{v}$ satisfies

$$
-\Delta_{q} \bar{v}=d(0) \bar{v}|\bar{v}|^{\delta-1} \quad \text { in } \quad R^{N} .
$$

Hence, as in the 1st case, we get a contradiction.

Proposition 4.3. Assume that $\left(H_{1}\right),\left(H_{2}\right),\left(H_{3}\right)$, and $\left(H_{4}\right)$ hold. Moreover, assume that one of the assumptions $\left(H_{s}\right)$ or $\left(H_{w}\right)$ holds. Then, the family of operators $\left(T_{\tau}\right)_{\tau \geq 0}$ satisfies the following properties:

$\left(P_{1}\right) \exists \tau_{0}>0$ such that, if $T_{\tau}$ has a fixed point $(u, v)_{\tau}$ for some $\tau \geq 0$ then $\tau \leq \tau_{0}$.

$\left(P_{2}\right) \exists C_{0}>0$ such that, if $(u, v)_{\tau}$ is a fixed point of $T_{\tau}$ with $\tau \leq \tau_{0}+1$, then $\left\|(u, v)_{\tau}\right\| \leq C_{0}$.

Proof: First, from the Maximum Principle, it follows that the problem

$$
(u, v)=T_{\tau}((u, v))
$$

is equivalent to find positive solutions $u, v$ of the following system

(4.74) $-\left(r^{N-1}\left|u^{\prime}(r)\right|^{p-2} u^{\prime}(r)\right)^{\prime}=r^{N-1}\left[a(r)|u(r)|^{\alpha}+b(r)|v(r)+\tau|^{\beta}\right]$,

(4.75) $-\left(r^{N-1}\left|v^{\prime}(r)\right|^{q-2} v^{\prime}(r)\right)^{\prime}=r^{N-1}\left[c(r)|u(r)|^{\gamma}+d(r)|v(r)|^{\delta}\right]$,

$$
u^{\prime}(0)=v^{\prime}(0)=u(R)=v(R)=0 .
$$

It follows that $0 \leq u(r), 0 \leq v(r)$ and by integrating on $[0, r]$ we obtain

$$
\begin{aligned}
& -u^{\prime}(r) \geq C r^{\frac{1}{p-1}}(v(r)+\tau)^{\frac{\beta}{p-1}}, \\
& -v^{\prime}(r) \geq C r^{\frac{1}{q-1}}(u(r))^{\frac{\delta}{q-1}} .
\end{aligned}
$$

Thus from (4.77)

$$
-u^{\prime}(r) \geq C r^{\frac{1}{p-1}} \tau^{\frac{\beta}{p-1}} .
$$


By integrating (4.79) from 0 to $R$, we obtain that

$$
u(0) \geq C R^{\frac{p}{p-1}} \tau^{\frac{\beta}{p-1}} .
$$

Now we suppose that $\left(P_{1}\right)$ is not true. Then there is a sequence $\left\{\tau_{n}\right\}$ such that

$$
\lim _{n \longrightarrow+\infty} \tau_{n}=+\infty
$$

and such that for each $\tau_{n}$ there exists a solution $\left(u_{n}, v_{n}\right)$ of $(4.74)-(4.76)$.

Then from (4.80) and (4.81), we have

$$
\lim _{n \longrightarrow+\infty}\left\|\left(u_{n}, v_{n}\right)\right\|=+\infty .
$$

Hence, respectively if $\left(H_{s}\right)$ [or $\left(H_{w}\right)$ ] holds then from Proposition 4.1 [Proposition 4.2] we obtain a contradiction.

We argue similarly if $\left(P_{2}\right)$ is not true.

Then for all $n \in \mathbf{N}$ there exists $\tau_{n} \leq \tau_{0}$ and a solution $\left(u_{n}, v_{n}\right)$ of (4.74)-(4.76) such that $\left\|\left(u_{n}, v_{n}\right)\right\|>n$, this implies that

$$
\lim _{n \longrightarrow+\infty}\left\|\left(u_{n}, v_{n}\right)\right\|=+\infty \text {. }
$$

Hence, from Proposition 4.1, and Proposition 4.2 we deduce a contradiction and Proposition 4.3 is proved.

\section{Proof of the theorems}

Proposition 5.1. We assume $\left(H_{1}\right),\left(H_{2}\right)$ and $\left(H_{3}\right)$. Then there exists a $\rho_{1}>0$ such that:

$\forall \rho \in\left[0, \rho_{1}[\right.$ and $\forall \lambda \in[0,1]$, we have that $(0,0)$ is the only fixed point of $S_{\lambda}$, in $B(0, \rho)$.

Proof: Let us take $\lambda \in[0,1]$ and $(u, v) \in \chi$ such that

$$
(u, v)=S_{\lambda}(u, v)
$$

with $\|(u, v)\|=\rho>0$. Notice that by the definition of $S_{\lambda}$ we get $u^{\prime} \leq 0$, $v^{\prime} \leq 0$ in $[0, R]$. By integrating on $[0, R]$ we have

(5.2) $u(0)=\lambda^{\frac{1}{p-1}} \int_{0}^{R}\left[t^{1-N} \int_{0}^{t} s^{N-1}\left(a(s)|u(s)|^{\alpha}+b(s)|v(s)|^{\beta}\right) d s\right]^{\frac{1}{p-1}} d t$

(5.3) $v(0)=\lambda^{\frac{1}{q-1}} \int_{0}^{R}\left[t^{1-N} \int_{0}^{t} s^{N-1}\left(c(s)|u(s)|^{\gamma}+d(s)|v(s)|^{\delta}\right) d s\right]^{\frac{1}{q-1}} d t$. 
Hence $\|(u, v)\|=u(0)+v(0)$. Thus, from $\left(H_{3}\right)$ there exist two numbers $l>0$ and $k>0$ such that

$$
\frac{\beta}{p-1}>\frac{l}{k}>\frac{q-1}{\gamma}
$$

Denote

$$
\sigma=(u(0))^{\frac{1}{l}}+(v(0))^{\frac{1}{k}},
$$

then $\|(u, v)\|<\sigma^{l}+\sigma^{k}$ and from (5.2) and (5.3) we deduce,

$$
\begin{aligned}
& (u(0))^{\frac{1}{l}} \leq C \lambda^{\frac{1}{l(p-1)}}\left[\sigma^{l \alpha}+\sigma^{k \beta}\right]^{\frac{1}{l(p-1)}}, \\
& (v(0))^{\frac{1}{k}} \leq C \lambda^{\frac{1}{k(q-1)}}\left[\sigma^{l \gamma}+\sigma^{k \delta}\right]^{\frac{1}{k(q-1)}} .
\end{aligned}
$$

Summing up (5.6) and (5.7), we deduce that $\sigma$ satisfies

$$
\begin{aligned}
1 \leq C \lambda^{\frac{1}{l(p-1)}}\left[\sigma^{l(\alpha-p+1)}\right. & \left.+\sigma^{k \beta-l(p-1)}\right]^{\frac{1}{l(p-1)}} \\
& +C \lambda^{\frac{1}{k(q-1)}}\left[\sigma^{l \gamma-k(q-1)}+\sigma^{k(\delta-q+1)}\right]^{\frac{1}{k(q-1)}} .
\end{aligned}
$$

Hence, there exist $m_{1}>0, m_{2}>0$ and $C>0$ such that

$$
C<\lambda^{m_{1}} \sigma^{m_{2}} \leq \sigma^{m_{2}} \text {. }
$$

On the other hand, by definition of $\sigma$, there exists $m_{3}>0$ such that

$$
\sigma \leq 2 \rho^{m_{3}} .
$$

Then, from (5.9) and (5.10) it suffices to choose $\rho_{1}$ such that $2^{1+\frac{1}{m_{3}}} \rho_{1}=$ $C^{\frac{1}{m_{2} m_{3}}}$, to complete the proof of Proposition 5.1.

Proof of Theorem 1.1: From Proposition 4.3, it follows that for $\rho_{2}=$ $C_{0}+1$, the equation $(u, v)=T_{\tau}((u, v))$ with $(u, v) \in \partial B\left(0, \rho_{2}\right)$ has no solution for $\tau \in\left[0, \tau_{0}+1\right]$. Then, $\operatorname{deg}\left(I-T_{\tau}, B\left(0, \rho_{2}\right), 0\right)$ is well defined and by the property of topological degree we get that

$$
\operatorname{deg}\left(I-T_{\tau}, B\left(0, \rho_{2}\right), 0\right)=\text { constant, } \quad \forall \tau \in\left[0, \tau_{0}+1\right] .
$$

Moreover, since for $\tau_{1}=\tau_{0}+1$, the operator $T_{\tau_{1}}$ has no a fixed point in $B\left(0, \rho_{2}\right)$, we have

$$
\operatorname{deg}\left(I-T_{\tau_{1}}, B\left(0, \rho_{2}\right), 0\right)=0 .
$$

Then, it follows from (5.11), that

$$
\operatorname{deg}\left(I-T_{0}, B\left(0, \rho_{2}\right), 0\right)=\operatorname{deg}\left(I-T_{\tau_{1}}, B\left(0, \rho_{2}\right), 0\right)=0 .
$$

Moreover, from Proposition 5.1, for $\rho_{1}>0$ sufficiently small we have

$$
\operatorname{deg}\left(I-S_{\lambda}, B\left(0, \rho_{1}\right), 0\right)=\text { constant } \quad \forall \lambda \in[0,1] .
$$


Hence

$$
\operatorname{deg}\left(I-S_{1}, B\left(0, \rho_{1}\right), 0\right)=\operatorname{deg}\left(I-S_{0}, B\left(0, \rho_{1}\right), 0\right)=+1 .
$$

Then, since $S_{1}=T_{0}$ and from (5.13) and (5.15), by the excision property we obtain

$$
\operatorname{deg}\left(I-S_{1}, B\left(0, \rho_{2}\right) \backslash B\left(0, \rho_{1}\right), 0\right) \neq 0 .
$$

So, there is a fixed point $(u, v)$ of $S_{1}$. Hence we obtain the conclusions in Theorem 1.1.

Proof of Theorem 1.2: Using Proposition 4.3, the proof of Theorem 1.2 is similar as the proof of Theorem 1.1.

Acknowledgement. The author thanks professor François de Thélin for suggesting the study of this problem and for helpful comments.

\section{References}

[1] A. Ahammou, On the existence of bounded solutions of nonlinear elliptic systems, Submitted.

[2] L. Boccardo, J. Fleckinger and F. DE Thélin, Elliptic systems with various growth, in "Reaction diffusion systems" (Trieste, 1995), Lecture Notes in Pure and Appl. Math. 194, Dekker, New York, 1998, pp. 59-66.

[3] A. Castro AND A. Kurepa, Infinitely many radially symmetric solutions to a superlinear Dirichlet problem in a ball, Proc. Amer. Math. Soc. 101(1) (1987), 57-64.

[4] P. Clément, R. F. Manásevich and E. Mitidieri, Some existence and non-existence results for a homogeneous quasilinear problem, Asymptot. Anal. 17(1) (1998), 13-29.

[5] P. Clément, R. F. Manásevich and E. Mitidieri, Positive solutions for a quasilinear system via blow up, Comm. Partial Differential Equations 18(12) (1993), 2071-2106.

[6] G. V. R. De Figueiredo, Semilinear elliptic systems, Preprint?

[7] P. Felmer, R. F. Manásevich and F. de Thélin, Existence and uniqueness of positive solutions for certain quasilinear elliptic systems, Comm. Partial Differential Equations 17(11-12) (1992), 2013-2029.

[8] J. Fleckinger, J. Hernández And F. De Thélin, On maximum principles and existence of positive solutions for some cooperative elliptic systems, Differential Integral Equations 8(1) (1995), 69-85. 
[9] B. Gidas and J. Spruck, A priori bounds for positive solutions of nonlinear elliptic equations, Comm. Partial Differential Equations 6(8) (1981), 883-901.

[10] A. El Hachimi and F. DE ThÉLin, Infinité de solutions radiales pour un problème elliptique superlinéaire dans une boule, $C . R$. Acad. Sci. Paris Sér. I Math. 315(11) (1992), 1171-1174.

[11] E. Mitidieri and S. I. Pokhozhaev, Absence of positive solutions for systems of quasilinear elliptic equations and inequalities in $\mathbf{R}^{N}$, Dokl. Akad. Nauk 366(1) (1999), 13-17.

[12] E. Mitidieri, A Rellich type identity and applications, Comm. Partial Differential Equations 18(1-2) (1993), 125-151.

[13] E. Mitidieri, Nonexistence of positive solutions of semilinear elliptic systems in $\mathbf{R}^{N}$, Differential Integral Equations $\mathbf{9 ( 3 )}$ (1996), 465-479.

[14] L. A. Peletier and R. C. A. M. van Der Vorst, Existence and nonexistence of positive solutions of nonlinear elliptic systems and the biharmonic equation, Differential Integral Equations 5(4) (1992), 747-767.

[15] R. Soranzo, A priori estimates and existence of positive solutions of a superlinear polyharmonic equation, Dynam. Systems Appl. 3(4) (1994), 465-487.

[16] M. A. S. Souto, Sobre a existencia de solucões positivas para sistemas couerativos não lineare, Ph.D. Thesis, UNICAMP (1992).

[17] J. VÉLIN AND F. DE ThÉLIN, Existence and nonexistence of nontrivial solutions for some nonlinear elliptic systems, Rev. Mat. Univ. Complut. Madrid 6(1) (1993), 153-194.

Département des Mathématiques et Informatique

Faculté des Sciences

Université Cadi Ayyad

El Jadida, BP20

Maroc

E-mail address: ahammou@ucd.ac.ma

Primera versió rebuda el 28 de setembre de 2000 , darrera versió rebuda el 23 de febrer de 2001. 